\title{
THE INTERSTELLAR CONNECTION TO SOLAR SYSTEM BODIES
}

\author{
J. MAYO GREENBERG and JONIEK HAGE \\ Huygens Laboratorium, Rijksuniversiteit te Leiden, P.O. Box 9504, 2300RA Leiden, The Netherlands
}

(Received 25 September, 1990)

...although I have now arrived at what I believe to be the true solution of the case, I have no material proof of it. I know it is so, because it must be so, because in no other way can every single fact fit into its ordered and recognized place. A. Christie, 1935

\section{Introduction}

It is now well recognized that interstellar signatures exist in such solar system bodies as comets (Greenberg and Hage, 1990) and meteorites (Clayton, 1988) but the story told by these identifications is far from complete. Ultimately one would like to be able to infer what the protoplanetary nebula conditions were at the time the bodies formed. This involves retracing the sequence starting with a dense interstellar cloud, through the accretion disk phase of the protoplanetary nebula to the various steps in the aggregation processes and finally to the physical and chemical metamorphic processes in the final body during the subsequent 4.55 billion years. Comets provide the best and most direct evidence for early (pre)solar system conditions. Fragments of comets in the form of interplanetary dust collected in the upper atmosphere of the Earth are not quite so directly related to the protoplanetary conditions. But - and this is important - they are directly accessible for laboratory studies which reveal details in chemical and isotopic composition on sub- $\mu \mathrm{m}$ scales (Bradley and Brownlee, 1986). Meteorites seem to have an advantage over the collected IDP's by providing many orders of magnitude more material available to study but, on the other hand, they are even further removed from their origins.

\section{The Interstellar Dust Model of Comets}

Let us first review the models of interstellar dust and comets we use here.

\subsection{INTERSTELLAR DUST IN THE PROTOSOLAR NEBULA}

There are basically two stages of interstellar dust (Greenberg, 1988): (1) Diffuse cloud dust in which volatiles (like $\mathrm{H}_{2} \mathrm{O}$ ) are not present; (2) Molecular cloud dust - of which protosolar nebula dust is a special advanced state - in which volatile icy mantles of $\mathrm{H}_{2} \mathrm{O}, \mathrm{CO}$ etc. are observed. The diffuse cloud dust consists of three 
populations. The major population, in terms of mass, consists of elongated particles with a silicate core and an organic refractory mantle, a few tenths of micrometers in size (i.e. core-mantle particles, Fig. 1a). The organic refractory material originally started out as ices of simple chemical compounds, but has undergone up to billions of years of ultraviolet photo-processing in interstellar space. The photoprocessing has changed the icy mixture into a carbon-rich and oxygen-poor refractory material containing many different organic molecules. The second population contains very small carbonaceous particles with radii smaller than $0.01 \mu \mathrm{m}$. Equally small silicate particles were originally proposed for the third population by Greenberg, but recent developments indicate that this population may consists of PAH molecules instead (see e.g. Leger et al., 1987). In the protosolar molecular cloud the dust consists of the coremantle particles with an additional outer mantle of volatile ices dominated by $\mathrm{H}_{2} \mathrm{O}$ (Fig. 1b). The expected mass fractions of three components of this dust are $0.20,0.19$ and 0.55 , for the silicate, organic refractory and the volatile material, respectively. These proportions are based on the model of interstellar dust extrapolated to the protosolar nebular cloud stage and constrained by the solar system abundances of the elements. In a very dense region all the smaller particles could be imbedded in the icy mantles. The mass fraction of carbonaceous particles is about 0.06 . The consideration of the volatile fraction assumes that the temperature in the protosolar nebula was not high enough to cause significant evaporation.

\subsection{THE COMET NUCLEUS AND COMA DUST}

Regarding the formation of comets, it has been proposed by Greenberg (1977, 1982, 1986) that initially aggregates of the protosolar nebula particles were made. Their tangled structure, like a bird's nest (Greenberg and Gustafson, 1981) is suggested as a possible way to provide them with rigidity. This structure may also be conjectured to be the result of random aggregation modified by slippage after particle collision at speeds of about $0.1 \mathrm{~km} / \mathrm{s}$. These aggregates in turn coalesce into larger bodies and ultimately comets. When a comet comes close to the sun, individual aggregates of various sizes are lifted from the comet and are exposed to the solar radiation. The ice mantles evaporate and the small carbonaceous particles are lost from the aggregates as well. Some of the organic material may also evaporate. Hereafter the aggregates consist of core-mantle particles composed only of organic refractory material and interstellar silicates as illustrated schematically in Fig. 1c. It is assumed that under these conditions this type of porous particle constitutes the coma dust in all but the innermost part of the coma. This assumption immediately implies that the 3.4 and 9.7 $\mu \mathrm{m}$ spectral features characteristic of interstellar dust, should show up in the infrared coma emission of a comet under appropriate conditions (Greenberg and Hage, 1990). The porosity $\mathrm{P}$ of an aggregate of interstellar dust is defined as

$$
\mathrm{P}=1-\left(\mathrm{V}_{\text {solid }} / \mathrm{V}\right)
$$


where $\mathrm{V}$ refers to the total volume of space taken up by the aggregate and $\mathrm{V}_{\text {solid }}$ is the volume of the solid material in the aggregate. The comet model predicts $0.9<\mathrm{P}<0.975$ for the coma dust and $0.6<\mathrm{P}<0.83$ for the porosity of comet nuclei.

\section{Comets}

Before the last return of comet Halley it had been customary to divide comets into dust and gases with the dust being thought of as various rocky constituents and the gases being mostly $\mathrm{H}_{2} \mathrm{O}$ with traces of other constituents (Whipple, 1990). A critical change in thinking took place when the results from VEGA-1 and -2 and GIOTTO showed that a substantial fraction of the dust carried organic matter containing the elements $\mathrm{O}, \mathrm{C}$, $\mathrm{N}$ and $\mathrm{H}$ which were previously associated only with volatiles (Kissel et al., 1986a,b; Jessberger et al., 1986). Although a connection between interstellar chemistry and comets had been suggested based largely on comparing interstellar molecules and

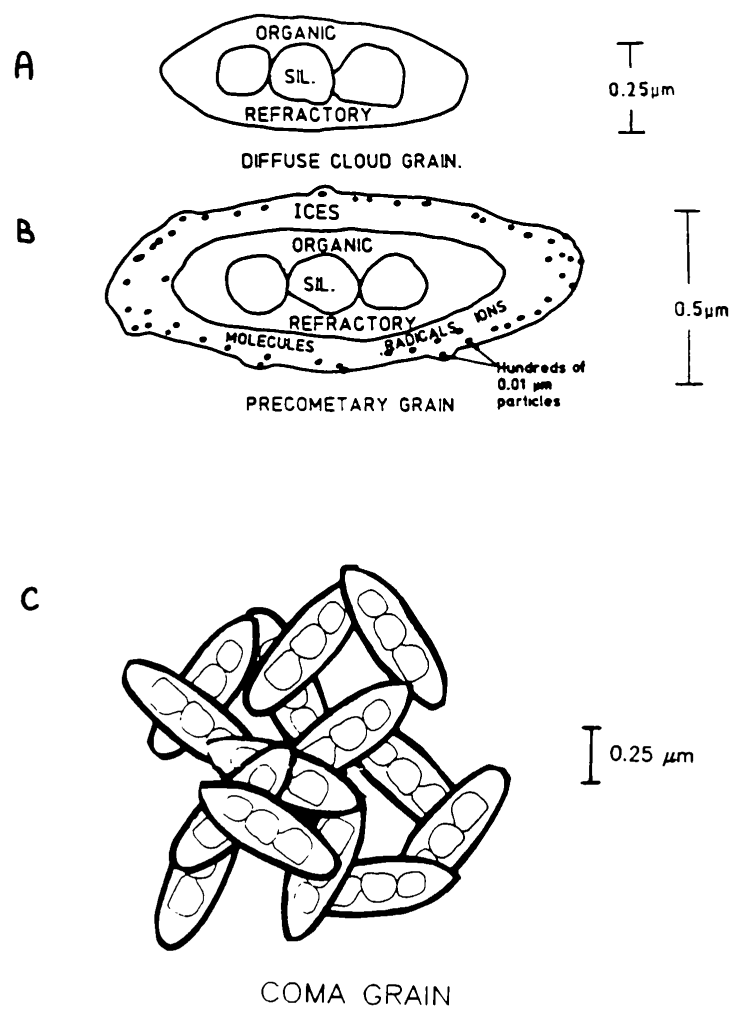

Fig.1. (a) Schematic of an interstellar dust grain which contains a core of silicates and a mantle of refractory organic material. (b) An interstellar grain after accretion of gases on its surface. Such grains make up a comet. (c) Schematic of a coma grain according to the interstellar dust model. It would have a porosity between 0.93 and 0.98 . 
cometary molecules (Delsemme, 1982) it had also been suggested that the interstellar dust is the carrier of interstellar chemistry to the comets for the dust as well as the gas (Greenberg, 1977, 1982, 1986).

\subsection{COMETARY DUST}

The solid fraction of comet Halley has been analyzed by mass spectroscopy (Kissel $e t$ al., 1986a,b; Kissel and Krueger, 1987), infrared emission (Hanner, 1987), and impact detectors (McDonnell et al., 1987, 1989; Mazets et al., 1987). Quantitative calculations (Greenberg and Hage, 1990) show that, in order to satisfy simultaneously such independent properties of the coma dust as the $3.4 \mu \mathrm{m}$ emission, the $9.7 \mu \mathrm{m}$ emission - shape as well as intensity, the mass distribution, and the composition, the coma dust must possess three main characteristics:

- its porosity is about $95 \%$ or higher;

- the principal aggregation units are silicate-core-organic-refractory mantle particles;

- the size of the core-mantle particles is about $0.1 \mu \mathrm{m}$.

The size and the core-mantle structure of the units are characteristic of the interstellar dust as it appears before entering the molecular cloud phase leading to the protoplanetary nebula. The ultraviolet processing of icy mantle mixtures that leads to the formation of the organics in interstellar space has been demonstrated by laboratory experiments. The organic mantles have been observed as providing a $3.4 \mu \mathrm{m}$ absorption in diffuse (non-molecular) clouds (Butchart et al., 1986, Adamson et al., 1990).

Although the rocky component of comet dust has been identified with various mixtures of anhydrous and hydrated silicates based on infrared spectra (Sandford, 1988), the evidence for hydrated silicates appears weak. Since the production of hydrated silicates indicates the past existence of liquid water it requires that at some time the comet was heated by some internal source to a rather high temperature - the solar radiation would never have been sufficient. If ${ }^{26} \mathrm{Al}$ was abundantly present in the comet during formation this could have occurred but it is an unlikely possibility statistically and no direct evidence of ${ }^{26} \mathrm{Mg}$ has been established in comets to justify this hypothesis (Jessberger $e t a l ., 1989$ ). The question of the degree of crystallinity of the silicates is not yet resolved but the data appear consistent with $90-95 \%$ being amorphous with a varying amount of crystallinity in different comets (Greenberg et al., 1989).

\subsection{Cometary gas}

The principal volatile constituent of comets is $\mathrm{H}_{2} \mathrm{O}$. Where does it come from? Interstellar dust as a source of all the $\mathrm{H}_{2} \mathrm{O}$ is a reasonable hypothesis. In molecular clouds $\mathrm{H}_{2} \mathrm{O}$ is generally the most abundant mantle constituent with $\mathrm{CO}$ running a fair second but down by about an order of magnitude (Grim and Greenberg, 1987). The question of whether $\mathrm{H}_{2} \mathrm{O}$ was evaporated from the dust because of heating in the nebula is answered differently by different theories about the protosolar nebula; that of 
Völk (1991) would seem to imply that it is not evaporated. Independent evidence for this may be the presence of $S_{2}$ as a parent molecule in comets (A'Hearn et al., 1983), but CS which had been predicted (Grim and Greenberg, 1987) has not been found (A'Hearn, 1990). On the other hand, an almost equally strong basis for attributing $\mathrm{H}_{2} \mathrm{O}$ in comets directly to interstellar dust and not to processes in the protosolar nebula is the abundance of $\mathrm{CO}_{2}$ in comets. The fractional amount of $\mathrm{CO}_{2}$ predicted by solar nebula chemistry is orders of magnitude lower than observed in comets (Anders, 1991). On the other hand, $\mathrm{CO}_{2}$ is predicted to be produced abundantly in interstellar dust by laboratory studies of UV processing of $\mathrm{H}_{2} \mathrm{O}: \mathrm{CO}$ mixtures and now has been observed in interstellar dust (d'Hendecourt and Jourdain de Muizon, 1990). According to A'Hearn (1990), the ubiquitous presence of $\mathrm{CO}_{2}$ in comets may only be deduced from $\mathrm{CO}_{2}+$ because in only one (or a few) cases $\mathrm{CO}_{2}$ is directly observed. Similarly, the fraction of $\mathrm{CH}_{4}$ in comets appears inconsistent with solar nebula chemical formation (Larson et al., 1989). For the other simple volatiles the relative abundances are consistent with interstellar dust in the condensed stage as well. The theoretical and laboratory work as well as astronomical high resolution observations have so far only hinted at the complete story of this dust composition but in broad terms it appears to be justified (Table I).

Finally, the over-abundance of CO in the comet coma (Eberhardt et al., 1987) and

TABLE I

Molecules directly observed in interstellar grains and/or strongly inferred from laboratory spectra and theories of grain mantle evolution

\begin{tabular}{lcc}
\hline molecule & \multicolumn{2}{c}{ Comment* } \\
\hline $\mathrm{H}_{2} \mathrm{O}$ & $\mathrm{O}$ & $\mathrm{M} 2$ \\
$\mathrm{CO}$ & $\mathrm{O}$ & $\mathrm{M} 2$ \\
$\mathrm{H}_{2} \mathrm{~S}$ & $\mathrm{O}$ & $\mathrm{M} 2$ \\
$\mathrm{NH}_{3}$ & $\mathrm{O}$ & $\mathrm{M} 2$ \\
$\mathrm{H}_{2} \mathrm{CO}$ & $\mathrm{O}$ & $\mathrm{M} 2$ \\
$\left(\mathrm{H}_{2} \mathrm{CO}\right)_{\mathrm{n}}$ & $\mathrm{I}$ & $\mathrm{M} 2$ \\
$\mathrm{OCN}^{-}$ & $\mathrm{O}$ & $\mathrm{M} 2(\mathrm{M} 1)$ \\
$\mathrm{NH}_{4}{ }^{+}$ & $\mathrm{O}$ & $\mathrm{M} 2$ \\
$\mathrm{CH}_{3} \mathrm{OH}$ & $\mathrm{O}$ & $\mathrm{M} 2$ \\
$\mathrm{OCS}^{2}$ & $\mathrm{O}$ & $\mathrm{M} 2$ \\
$\mathrm{CO}_{2}$ & $\mathrm{I}$ & $\mathrm{M} 2$ \\
$\mathrm{CH}_{4}$ & $\mathrm{I}$ & $\mathrm{M} 2$ \\
$\mathrm{~S}_{2}$ & $\mathrm{I}$ & $\mathrm{M} 2$ \\
complex organic & $\mathrm{O}$ & $\mathrm{M} 1$ \\
Silicate & $\mathrm{O}$ & $\mathrm{C}, \mathrm{B}$ \\
Carbonaceous & $(\mathrm{O}, \mathrm{I})$ & $\mathrm{B}$ \\
\hline
\end{tabular}

*O=observed; $\mathrm{I}=$ inferred; $\mathrm{M} 1=$ inner mantle, M2 = outer mantle; $\mathrm{B}=$ small bare, $\mathrm{C}=$ core 
the presence of $\mathrm{CN}$ and other molecular fragments (A'Hearn et al., 1986) are to be attributed not to the initial volatile fraction of comets but rather to the organic component. It is to be noted that what is considered refractory in the interstellar medium at temperatures less than $100 \mathrm{~K}$ or, in the laboratory at $300 \mathrm{~K}$, will readily evaporate at solar system temperatures. The temperatures of porous comet dust for masses less than $\approx 10^{-10} \mathrm{~g}$ is well over $400 \mathrm{~K}$ at $1 \mathrm{AU}$ and a significant fraction of the organics is expected (and is observed from mass spectra) to have evaporated. It is this component which must be photo-dissociated to provide the observed gases such as $\mathrm{CO}$ and $\mathrm{CN}$. Incidentally, if the dust were not porous it would not get hot enough and it would also not have enough surface area from which these extra volatile fractions could evaporate.

\section{Meteorites}

The question was raised at this meeting (Wasserburg, informal discussion) why carbonates exist in meteorites. It is tempting to suggest that they may be attributed to $\mathrm{CO}_{2}$ in interstellar dust if the dust aggregated cold enough to have preserved it in the grain mantles along with the $\mathrm{H}_{2} \mathrm{O}$. The obvious implication of this is that after aggregation of the parent body there was enough heating to melt the water. The evidence for liquid $\mathrm{H}_{2} \mathrm{O}$ in the parent bodies of carbonaceous chondrites allows for the dissolving of $\mathrm{CO}_{2}$ in water and the subsequent formation of carbonates. This suggestion, however, raises the question of why the organic carbon component of carbonaceous meteorites is at least ten times less than that in comets because, if $\mathrm{H}_{2} \mathrm{O}$ was preserved, the organics should certainly also have been preserved. Isotopic fractionation in pre-aggregated interstellar dust from gas phase and solid state (photo-) chemistry may ultimately provide an answer to this question.

\section{Other Solar System Bodies}

Are there other solar system bodies which will reveal signatures of interstellar dust and gas chemistry? First of all, there is every reason to believe that between comets and asteroids there may be many intermediate types of bodies. While cometary debris is believed to be more randomly oriented in orbits than asteroidal debris, there is some evidence from the optical properties of the zodiacal light particles that a significant contribution to the out-of-ecliptic particles comes from asteroidal material (LevasseurRegourd, 1990).

\section{Future Needs}

Direct access to original solar system bodies like comets, asteroids, and the satellites of outer planets will ultimately be required to probe with complete reliability the origin of the solar system. Until that occurs the aim should be to use available material in the 
form of interplanetary dust and meteorites for laboratory studies and to fully exploit remote sensing. Although interstellar dust is not yet in hand it is important to continue analog ultraviolet photo-processing laboratory studies on its chemical evolution along with theoretical studies of the interactions of molecules in the gas and on dust surfaces. It should be noted that even when probes to solar system bodies bring back data and material, we still will have to provide a theoretical and laboratory basis for interpreting the properties of this material.

Even the laboratory produced mixtures of complex organic material are difficult to fully identify (Agarwal et al., 1985). Since laboratory residues are now and in the near future the most easily available material representing the original interstellar organics, they should serve as a proving ground for recovered matter from real space bodies. Molecules in the laboratory can be taylored to fit techniques for baseline studies. Another advantage of the laboratory studies over direct solar body material studies is that arbitrary isotope enrichment may be used to assist in identifications. A large number of laboratory experiments suggest themselves based on comet Halley results alone. For example, we should investigate the volatile fraction of laboratory produced organic residues at solar system temperatures. Testing new and old analytical techniques on laboratory organics is a must because reliability and consistency have to be controlled. A major effort should be directed to develop smaller and smaller spatial resolution in sample analysis because the fineness of the observed structures are themselves a probe of initial formation mechanisms. Finally, with respect to material recovery from solar system bodies by space vehicles, the preservation of morphological structure is of prime importance. With the burgeoning evidence for the fluffy structure of comets on a sub- $\mu \mathrm{m}$ level and the information it imparts on the chemical evolution of the early and pre-solar system, the preservation of fragile material should be a prime aim in the future comet nucleus sample return mission, ROSETTA.

\section{Acknowledgement}

We would like to acknowledge partial support for this research from NASA grant \#NGR33018148.

\section{References}

Adamson, A. J., Whittet, D. C. B., and Duley W. W.: 1990, MNRAS 243, 400-404.

Agarwal, V. K., Schutte, W., Greenberg, J. M., Ferris, J. P., Briggs, R., Connor, S., Van de Bult, C.P.E.M., and Baas, F.: 1985, Origins of Life 16, 21.

A'Hearn, M. F., Feldman, P. D., and Schleicher, D. G.: 1983, Astrophys. J. 274, L99

A'Hearn, M. F., Hoban. S., Brich, P. V., Bowers, C., Martin, R., and Klinglesmith III, D. A.: 1986, Nature 324, 649.

A'Hearn, M. F.: 1990, private communication.

Anders, E.: 1991, in E.K. Jessberger (ed.) Analysis of Samples from Solar System Bodies Space Science Reviews, this volume.

Bradley, J. R. and Brownlee, D. E.: 1986, Science 231, 1542. 
Butchart, I., McFadzean, A. D., Whittet, D. C. B., Geballe, T. R., and Greenberg, J. M.: 1986, Astr. Ap. 154, L5.

Clayton, D.D.: 1988, in M. E. Bailey, D. A. Williams (eds.) Dust in the Universe, Cambridge Univ. Press, 145-151.

Delsemme, A.H.: 1982, in Wilkening, L. L. (ed.) Comets, University of Arizona Press, Tucson.

d'Hendecourt, L. B. and Jourdain de Muizon: 1989, Astr. Ap., 223, L5-L8.

Eberhardt, P., Krankowsky, D., Schulte, W., Dolder, U., Lämmerzahl, P., Berthelier, J. J., Woweries, J., Stubbemann, U., Hodges, R. R., Hoffman, J. H., and Illiano J. M.: 1987, Astron. Astrophys. 187, 481-484.

Greenberg, J. M.: 1977, in Delsemme, A. H. (ed.) Comets, Asteroids, Meteorites, Univ. of Toledo Press, 491.

Greenberg, J. M.: 1982, in Wilkening, L. L. (ed.) Comets, University of Arizona Press, Tucson, 131.

Greenberg, J. M.: 1986, in Lagerkvist, C. I., Lindblad, B. A., Lundstedt, H., and Rickman, H. (eds.) Asteroids, Comets and Meteors II, Uppsala University Press, 221.

Greenberg, J.M.: 1988, in Bailey, M. and Williams, D. A., (eds.) Dust in the Universe, Cambridge University Press 121.

Greenberg, J. M. and Gustafson, B.: 1981, Astr. Ap. 93, 35.

Greenberg, J. M., Zhao, N. S., and Hage, J. I.: 1989, Adv. Space Res 9, 3.

Greenberg, J. M. and Hage, J. I.: 1990, Ap. J. 321, 260.

Grim, R. J. A. and Greenberg, J. M.: 1987, Astr. Ap. 181, 155.

Hanner, M.S. Tokunaga, A.T., Golisch, W.E., Gripe, D.M., and Kaminski, C.D.: 1987, Astr. Ap. 187, 653.

Jessberger, E.K., Kissel, J., Fechtig, H., and Krueger, F.R.: 1986, in Proc. Comet Nucleus Sample Return, ESA-SP 249, 27.

Jessberger, E.K., Kissel, J., and Rahe, J.: 1989, in S.K. Atreya, J.B. Pollak, and M.S. Matthews (eds.) Origin and Evolution of Planetary and Satellite Atmospheres, The University of Arizona Press, Tucson, 167-191.

Kissel et al.: 1986a, Nature 321, 280-282.

Kissel et al.: 1986b, Nature 321, 336-338.

Kissel, J. and Krueger, F. R.: 1987, Nature 326, 755.

Larson, et al.: 1989, Ap. J. 338, 1106.

Leger, A., d'Hendecourt L. Boccara N. (eds.): 1987, Polycyclic Aromatic Hydrocarbons and Astrophysics, Reidel, Dordrecht.

Levasseur-Regourd, A-C.: 1990, Paper presented at IAU Colloquium 134, September, Kyoto, Japan.

Mazets E. P. et al.: 1987, Astr. Ap. 187, 699.

McDonnell, J. A. M. et al.: 1987, Astr. Ap. 187, 719.

McDonnell, J. A. M., Pankiewicz, G. S., Birchley, P. N. W., Green, S. F., and Perry, C. H.: 1989, in Workshop on Analysis of Returned Comet Nucleus Samples, Milpitas, California, in the press.

Sandford, S. A.: 1988 in M. E. Bailey, D. A. Williams (eds.) Dust in the Universe, Cambridge Univ. Press, 193-197.

Völk, H.J. and Morfill, G.: 1991, in E.K. Jessberger (ed.) Analysis of Samples from Solar System Bodies Space Science Reviews, this volume.

Whipple, F.L.: 1989, Ap. J. 341, 1. 